Introduction Gastric cancer is the 4th leading cause of cancer death worldwide. Prognosis remains poor, largely due to late diagnosis. Early gastric neoplasia carries a far better prognosis with a 5 year survival of $85 \%$. Traditionally, gastrectomy is the definitive locoregional treatment, but carries significant perioperative morbidity. For early gastric cancer without risk of nodal metastasis, endoscopic en bloc resection with endoscopic submucosal dissection (ESD) is a feasible alternative. We present a prospective cohort analysis of patients with early gastric neoplasia undergoing ESD at a single tertiary Centre in the United Kingdom

Methods Patients were all referred through local cancer networks after staging to exclude metastatic disease. Patients underwent gastroscopy with biopsy for histology and contrast computed tomography (CT) for staging. High definition endoscopy \& chromoendoscopy with indigo carmine were used to help delineate lesions. Pathology was confirmed by expert GI pathologists prior to resection. Surgery was discussed with all patients as an alternative to ESD. All gastric ESD were performed under propofol sedation by a single endosocopist with specialist training.

Results Over 24 months, 19 patients underwent ESD. Mean age was 71 years (range 23-87). 68\% were male. Two lesions (10\%) were in the proximal stomach, 1 in mid body (5\%) 16 in distal stomach (85\%). Mean resection size was $28 \mathrm{~mm}(15-58)$. Mean resection time was 71 mins from intubation. En-bloc resection was achieved in $15 / 19(74 \%)$ patients. $16 / 19$ patients ( $84 \%$ ) had a clear deep resection margin. Resection histology was high grade dysplasia in $52 \%$, carcinoma in situ 11\%, LGD 5\%, poorly differentiated adenocarcinoma $21 \%$, neuroendocrine tumour in $5 \% .13 / 16$ with clear resection margins post ESD remain in follow up. Of these 11/13 (87\%) remain free of disease at most recent biopsy (median follow up 6 months, range $0-38$ ). Both recurrences were LGD at the resection site. One was treated with laser ablation and the other with repeat ESD. There were no perforations or bleeding seen in our cohort during ESD

Conclusion In this small cohort of early gastric neoplasia, ESD appears to provide a safe and effective alternative to surgery with encouraging durability although our follow up time is short. Early recognition of lesions is essential to offer patients a minimally invasive curative intervention. With increasing use of high definition endoscopy more patients will become eligible for therapy. Long term durability remains to be seen.

Disclosure of Interest None Declared.

\section{PTH-046 ANALYSIS OF METACHRONOUS COLORECTAL ADENOMA SITES SUGGESTS PROXIMAL OCCURRENCE IS MORE PROBABLE}

doi:10.1136/gutjnl-2013-304907.533

1."R Rosser, 'B M Corfe, ${ }^{2} \mathrm{~K}$ S Chapple. 'Surgical Oncology, The University of Sheffield; ${ }^{2}$ Colorectal Surgery, Northern General Hospital, Sheffield, UK

Introduction A human sporadic colorectal adenoma may influence the formation of another adenoma even after its removal, with up to $60 \%$ of such patients developing metachronous adenomas following adenoma excision. However data regarding the occurrence site of a metachronous colorectal adenoma relative to the index adenoma is scarce. Therefore, we investigated whether a metachronous colorectal adenoma was more likely to occur in the same, proximal or distal segment as the index adenoma.

Methods A prospectively-maintained database was interrogated to identify all colonoscopies performed over a ten-year period at a single university teaching hospital. All patients undergoing polypectomy were cross-referenced against a histological database to confirm adenoma status. Patients with synchronous adenomas were excluded. The site of adenoma removal at index and subsequent colonoscopy was recorded and categorised into three groups (proximal, distal or same segment).

Results 15,121 colonoscopies and 4759 polyp events were recorded. 361 patients (235 male, 126 female, median age [range] 66 [32-91] years) developed a single metachronous adenoma at follow-up colonoscopy. Metachronous adenomas were more likely to develop in a different bowel segment (61\%, $95 \%$ c.i. $56 \%$ $>66 \%$ ) to that of the index adenoma (39\%, $95 \%$ c.i. $34 \%>44 \%$; $P<0.01$ one way Chi-squared test). Metachronous adenomas were more likely to occur at a site proximal $(41 \%, 95 \%$ c.i. $36 \%>$ $46 \%)$ to the index adenoma than either the same segment $(39 \%$, $95 \%$ c.i. $34 \%>44 \%)$ or a more distal segment $(20 \%, 95 \%$ c.i. $16 \%$ $>24 \% ; P<0.01$ one way Chi-squared test). Proximally-sited metachronous adenomas were more likely to occur in a segment further away (mean [SD] segments travelled 3.5 [2.3]) from the index adenoma than distally-sited metachronous adenomas (2.6 [1.8] segments travelled; $P<0.01$ Kruskal-Wallis one way ANOVA).

Conclusion A metachronous human sporadic colorectal adenoma is more likely to be found in a section of the colorectum proximal to that of the index adenoma. Travel in segments may be significant. Taken together, the data suggest research effort into underlying mechanisms of metachronous adenoma is needed.

Disclosure of Interest None Declared.

\section{PTH-047 A PROSPECTIVE STUDY ON THE IMPACT OF A 'BOWEL CLEANSING CARE BUNDLE' ON INPATIENT COLONOSCOPY VISUALISATION SCORES: A LONDON DISTRICT HOSPITAL EXPERIENCE}

doi:10.1136/gutjnl-2013-304907.534

1.*R K Fofaria, 'N Hamilton, 'V J Blackwell, 'D Sherman, 'A W Sharif. 'Gastroenterology Department, Central Middlesex Hospital, London, UK

Introduction It is recognised that a well prepared colon increases adenoma detection and removal rates ${ }^{1}$ and the discovery of other significant pathology at endoscopy. Several factors can lead to a suboptimal outcome at inpatient colonoscopy but inadequate bowel preparation is a major contributor ${ }^{2}$. Potentially, this leads to repeat and/or alternative procedures and costly and prolonged hospital length of stays. 'Care Bundles' improve outcomes by decreasing the unwanted variation in clinical care and ensure the application of accepted clinical practices ${ }^{3}$. Consequently, we created a bowel cleansing bundle to address these issues and see whether it would lead to better visualisation scores.

Methods All inpatients requiring colonoscopy were recruited over two six-month periods at a London district hospital. A bowel cleansing bundle was devised formalising accepted clinical guidelines and it was implemented for all subsequent inpatient colonoscopies during the latter study phase. The bundle included sections on timings for the administration of bowel preparation agents, cessation of anti-motility agents, nutrition and hydration. Visualisation scores were noted from the endoscopists' reports (good, moderate and poor) and patients were followed up until discharge.

Results In total there were 93 eligible procedures. 53 patients in the pre-bundle population [56\% Male, average age 69 years] and 40 patients in the post-bundle population [three exclusions, $57 \%$ male, average age 64 years] of which: 'good' visualisation scores $=45 \%$ vs $81 \%$, 'moderate' $=21 \%$ vs $1 \%$ and 'poor' $=34 \%$ vs $16 \%$. Implementation of the bundle led to significantly superior 'good' visualisation scores $(p=<0.001)$. 